\title{
Multi-Stage Schemes for the Euler and Navier-Stokes Equations with Optimal Smoothing
}

\author{
John F. Lynn* and Bram van Leer ${ }^{\dagger}$ \\ Department of Aerospace Engineering, \\ The University of Michigan, \\ Ann Arbor, MI 48109-2140
}

\begin{abstract}
The effect of a recently derived local preconditioning matrix [1] on discretizations of the spatial Euler operator is a strong concentration of the pattern of eigenvalues in the complex plane. This makes it possible to design multi-stage schemes that systematically damp most highfrequency waves admitted by the particular discrete operator.

The resulting schemes are not only preferable as solvers in a multi-grid strategy, they are also superior single-grid schemes, as the preconditioning itself already accelerates the convergence to a steady solution, and the high-frequency damping provides robustness.

In this paper, we describe the optimization technique, use it to obtain the optimal sequence of time-step values for upwind Euler discretizations, and present some convergence results for numerical integrations performed with the new schemes. Furthermore, the extension to discrete Navier-Stokes operators is treated.
\end{abstract}

\section{Introduction}

Explicit marching schemes for the Euler and NavierStokes equations must feature effective high-frequency damping in order to be suited for use in multi-grid marching. Multi-stage schemes offer the flexibility to achieve the desired smoothing properties. Until recently, however, the design of optimally smoothing multi-stage schemes was based entirely on the scalar one-dimensional $[2,3]$ or twodimensional [4] convection equation.

In [5] we presented, for the first time, a design approach for Euler schemes in which the Fourier transform of the full spatial operator is used. This approach has become possible owing to a breakthrough in preconditioning algorithms [1], reported at the 10th AIAA CFD Conference, Honolulu, June 1991. Presented

\footnotetext{
*Doctoral Candidate, Aerospace Engineering and Scientific Computation

†Professor, Associate Fellow AIAA
}

there was a local preconditioning matrix that removes the spread among the characteristic speeds as much as possible. It achieves what can be shown to be the optimal condition number for the characteristic speeds, namely, $1 / \sqrt{1-\min \left(M^{2}, M^{-2}\right)}$, where $M$ is the local Mach number. This is a major improvement over the condition number before preconditioning, which equals $(M+1) / \min (M,|M-1|)$.

The effect - of the preconditioning on discretizations of the spatial Euler operator is a strong concentration of the pattern of eigenvalues in the complex plane. This, finally, makes it possible to design multi-stage schemes that systematically damp most high-frequency waves admitted by the particular discrete operator. The design technique is an extension of the techniques used in $[3,6,4]$.

The resulting schemes are not only preferable as solvers in a multi-grid strategy, they are also superior single-grid schemes, as the preconditioning itself already accelerates the convergence to a steady solution, and the high-frequency damping provides robustness.

In this paper we describe the optimization technique, use it to obtain the optimal sequence of time-step values for upwind Euler discretizations, and present some convergence results for numerical integrations performed with the new schemes. Furthermore, the extension to discrete Navier-Stokes operators is treated. The Euler preconditioning matrix on which this research is based is an improvement over the matrix presented in [1]; the NavierStokes preconditioner is new. Both are presented in a companion paper on preconditioning, simultaneously submitted to this conference [7].

\section{Previous scalar analysis}

Tai's [3] procedure for optimizing the high-frequency damping in a one-dimensional convection scheme is a geometry exercise in the complex plane: putting the zeros of the multi-stage amplification factor on top of the locus of the Fourier transform - the "Fourier footprint" - of the discrete spatial operator. This can be achieved for one 
Fourier Footprint of $\kappa=1 / 3$

Six-Stage Scheme

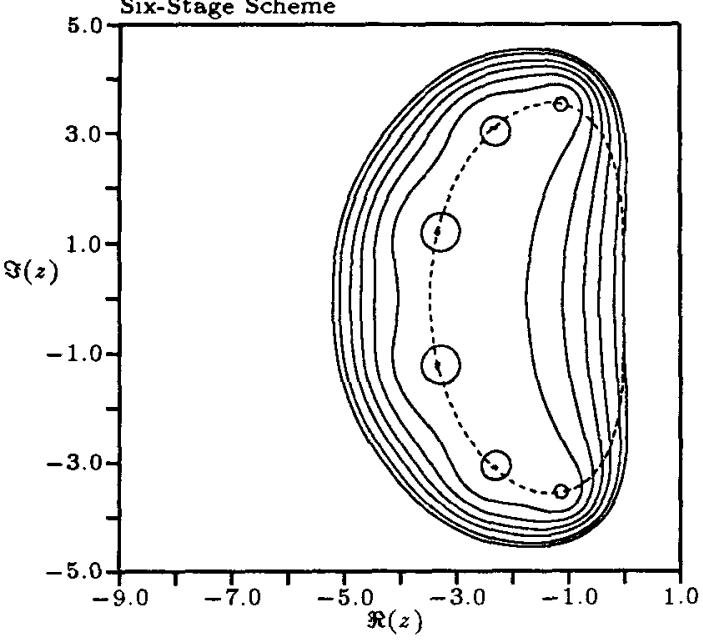

11.00

20.85

30.65

40.50

50.35

$\begin{array}{ll}6 & 0.20\end{array}$

80.01
Figure 1: Fourier footprint (dashed line) of the thirdorder upwind-biased spatial discretization of the onedimensional convection operator, and level lines (solid) of the amplification factor of Tai's optimal six-stage scheme.

specific value of the time-step, the finding of which is part of the design process. An example of the result of this procedure is shown in Figures 1 and 2.

Catalano and Deconinck [4] relaxed the condition that the zeros must lie exactly on the Fourier locus, thereby achieving a further reduction of the maximum amplification factor for the high frequencies.

For a two-dimensional discrete convection operator the Fourier footprint no longer is a single curve, but covers an area; the location and shape of this area vary greatly with the convection direction. Figures 3 and 4 show the locus for the first-order upwind-differencing operator, for convection directions of $10^{\circ}$ and $45^{\circ}$. The frequencies included in the footprint are $\beta_{x} \in[0, \pi], \beta_{y} \in[0, \pi]$. Highfrequency damping by a fixed multi-stage scheme (coefficients independent of flow direction) is easily achieved for modes propagating in the physical convection direction, but is fundamentally difficult for modes varying in the normal direction, especially if the convection is almost in the grid direction. This is what we may call the singlegrid alignment problem; its solution lies beyond the scope of this paper.

The alignment problem is evident in Figure 3 from the low-high frequency combinations found near the origin. To damp these, zeros must be put close to the origin; to benefit from these zeros, a large time-step would be needed; this works against numerical stability.

For two-dimensional convection Tai as well as Catalano and Deconinck use a one-dimensional optimization: they only consider high-frequency plane waves moving in the flow direction. Tai accepts the optimal sequence of time-step ratios for one-dimensional convection and merely adjusts the final Courant number; the latter de-

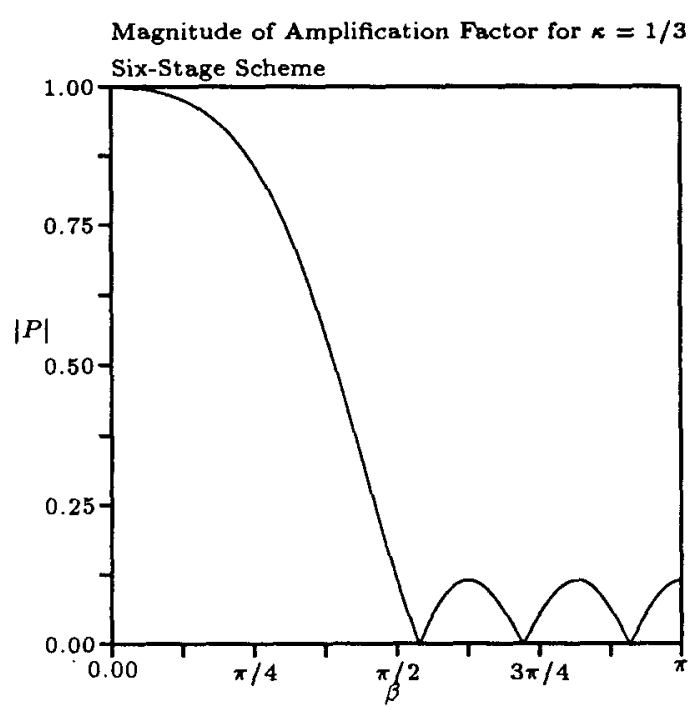

Figure 2: Modulus of the amplification factor as a function of spatial frequency, for the case of Figure 1.

pends on the flow angle. Catalano and Deconinck repeat the optimization for each flow angle; this makes all parameters dependent on the flow angle, which is less desirable. Moreover, the alignment problem causes the the parameters to vary strongly when the flow angle becomes small.

We have redone the optimization for two-dimensional convection using the full two-dimensional footprint. For small flow angles, though, we excluded the low-high frequency combinations which would render the optimization meaningless. The optimal coefficients derived using spatial operators associated with the two-dimensional convection equation are very close to the coefficients obtained with the Modified Roe operator for the preconditioned Euler equations. Figure 21 shows the Fourier footprint of the first-order upwind discretization (high-frequency component) for the two-dimensional convection equation superimposed upon level lines of the multi-stage scheme based upon the modified Roe operator for the preconditioned Euler equations. As can be seen in the figure, these coefficients are close to optimal for the two-dimensional convection discrete operator as well. It is important to make an appropriate choice of the length scale used in defining the Courant number $\nu$ (cf. Section 5).

\section{Euler equations: effect of pre- conditioning}

For the two-dimensional Euler equations the situation gets even worse, because there now are different kinds of physical signals propagating in all possible directions at different speeds; these are more or less accurately represented by the discrete operator and produce different concentrations of eigenvalues in its Fourier footprint. Figures 5 to 8 show the Fourier footprint of the first-order up- 


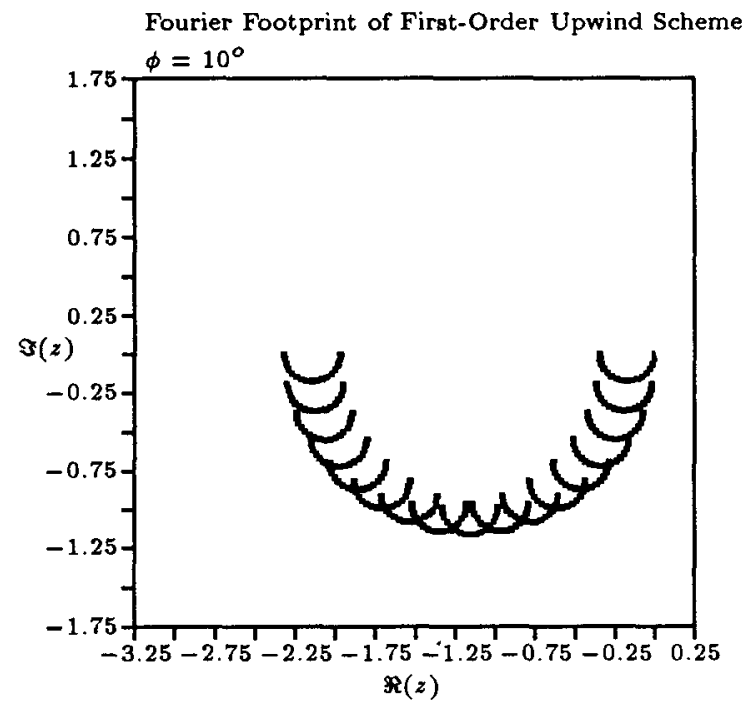

Figure 3: Fourier footprint of the first-order upwind approximation of the two-dimensional convection operator; convection angle $\phi=10^{\circ}$.

wind scheme for the Euler equations, based on Roe's [8] upwind-biased flux formula, for a range of Mach numbers. All figures are for the case when the flow speed is aligned with the grid.

The different sizes of the different concentrations in the footprint make it impossible to place the zeros of a multi-stage amplification factor at fixed locations in the complex plane and still achieve good high-frequency damping for all Mach numbers (even disregarding flow angles).

The next sequence of figures, 9 to 12 , show the Fourier footprint of the preconditioned first-order upwind scheme for the Euler equations, again based on Roe's flux formula, for a range of Mach numbers. The frequencies included in the footprints are $\beta_{x} \in[0, \pi], \beta_{y} \in[0, \pi]$. The preconditioning matrix is the one presented in [1]. A comparison with the previous sequence shows that removing the variation among the characteristic convection speeds has resulted in a thorough clean-up of the footprint. Especially impressive is the job it does for small $M$. For $M \uparrow 1$ a growing separation of two regions of concentration of eigenvalues is observed; this corresponds to the growing disparity between the acoustic speeds in the flow direction ( $=q \sqrt{1-M^{2}}, q \equiv$ flow speed) and normal direction $(=q)$. For $M>1$ the footprint starts looking very much like one for scalar convection (cf. Figure 3); this is because all signals in supersonic flow move downstream.

\section{Optimization procedure}

The procedure for optimizing high-frequency damping aims at minimizing the maximum of the modulus of the scheme's amplification factor over the set of highfrequency eigenvalues. The input parameters are the time-

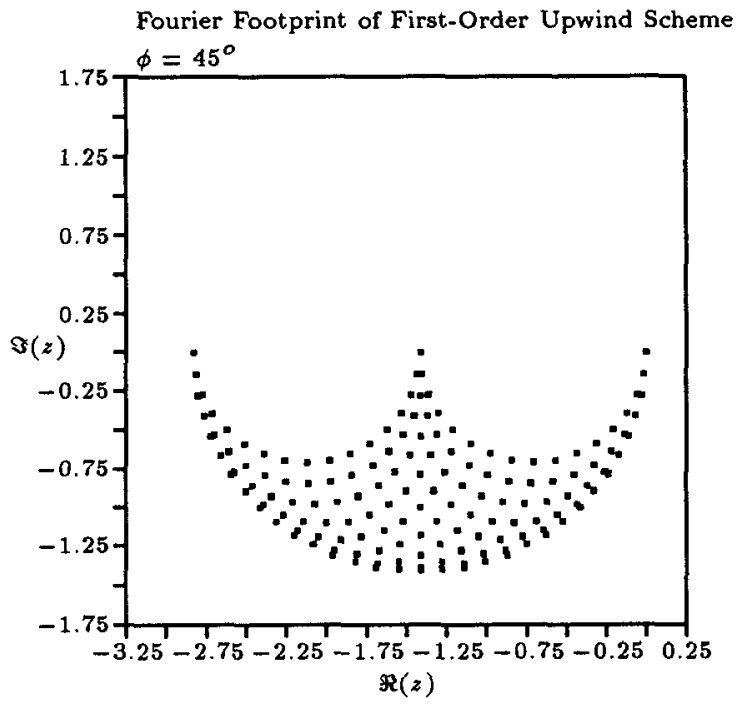

Figure 4: Fourier footprint of the first-order upwind approximation of the two-dimensional convection equation; convection angle $\phi=45^{\circ}$.

step values $\Delta t^{(k)}, k=1, \ldots, m$, of an $m$-stage algorithm. When updating the solution of

$$
U_{t}=\operatorname{Res}(U)
$$

from time level $t^{n}$ to $t^{n+1}=t^{n}+\Delta t$, the method takes the form

$$
\begin{aligned}
U^{(0)} & =U^{n} \\
U^{(k)} & =U^{(0)}+\Delta t^{(k)} \operatorname{Res}\left(U^{(k-1)}\right), k=1, . ., m,(3) \\
U^{n+1} & =U^{(m)}
\end{aligned}
$$

with $\Delta t=\Delta t^{(m)}$. According to linear theory, one step with the full scheme multiplies each eigenvector of the operator $\operatorname{Res}(U)$, with associated eigenvalue $\lambda$, by a factor of the form

$$
P(z)=1+z+\sum_{k=2}^{m} c_{k} z^{k}
$$

where

$$
z=\lambda \Delta t
$$

generally is complex. The $m-1$ coefficients $\alpha_{k}$ relate to the time-step ratios $\alpha_{k}=\Delta t^{(k)} / \Delta t$; the actual time step $\Delta t$ is the $m$ th parameter.

The optimization procedure starts out by computing, for a fixed combination of $M$ and $\phi$ ( $\equiv$ flow angle), a discrete set of eigenvalues for wave-number pairs $\left(\beta_{x}, \beta_{y}\right)$ in the high-frequency range, i.e.

$$
\left|\beta_{x}\right| \in\left(\frac{\pi}{2}, \pi\right) \text { and/or }\left|\beta_{y}\right| \in\left(\frac{\pi}{2}, \pi\right) \text {. }
$$

Assuming a set of starting values for the $m$-stage scheme, for instance Tai's values, the value of $|P(z)|$ is computed for all eigenvalues previously obtained, and its maximum 


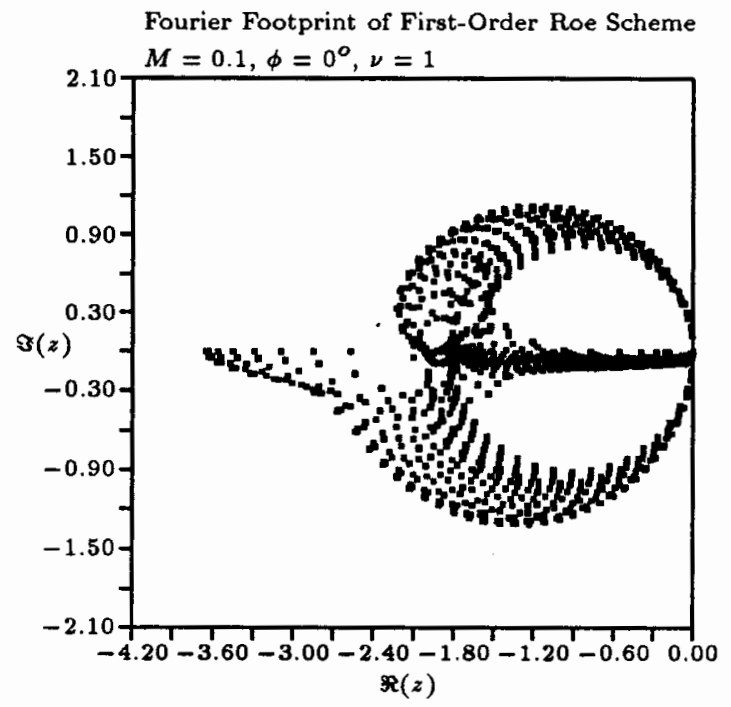

Figure 5: Fourier footprint of the first-order upwind approximation of the spatial Euler operator, for $M=0.1$, and flow angle $\phi=0^{\circ}$. The time-step chosen corresponds to a Courant-number value of 1 .

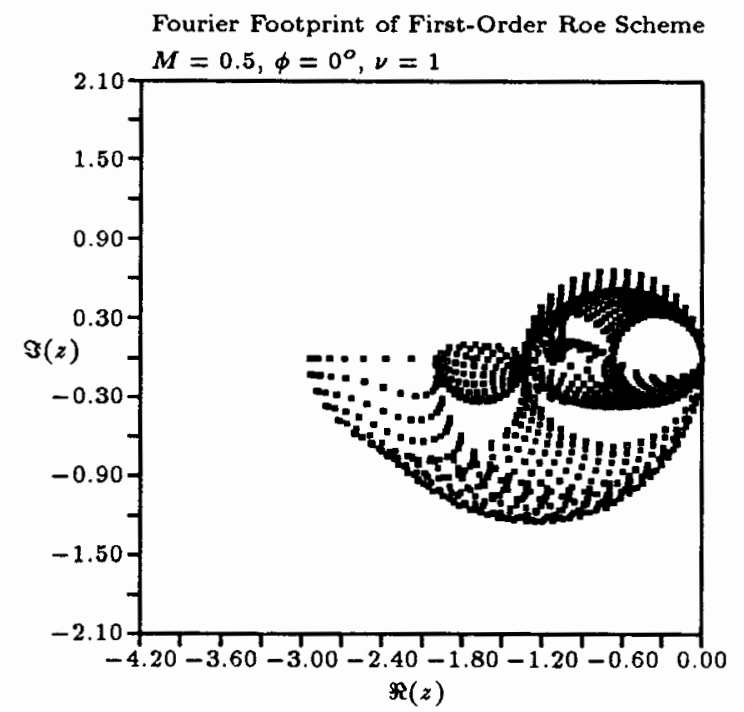

Figure 6: Fourier footprint of the first-order upwind approximation of the spatial Euler operator, for $M=0.5$, and flow angle $\phi=0^{\circ}$.

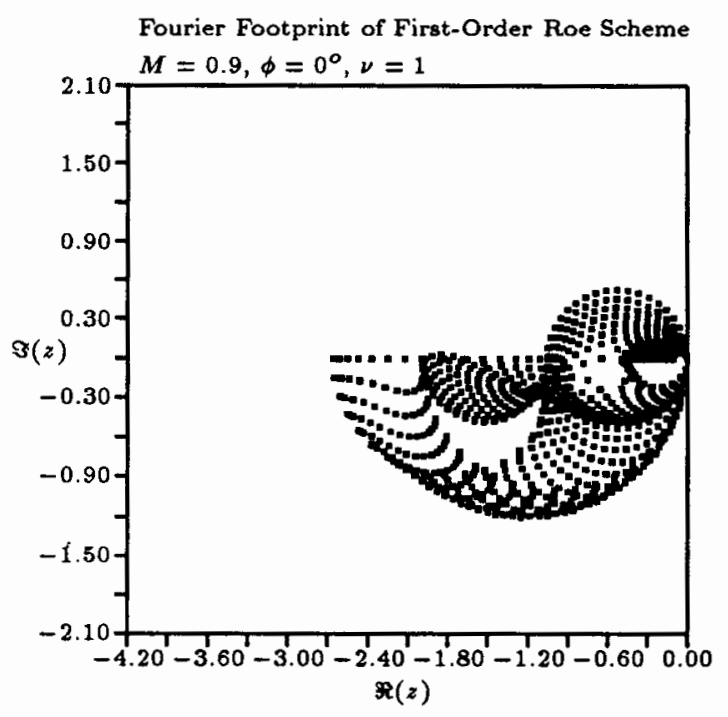

Figure 7: Fourier footprint of the first-order upwind approximation of the spatial Euler operator, for $M=0.9$, and flow angle $\phi=0^{\circ}$.

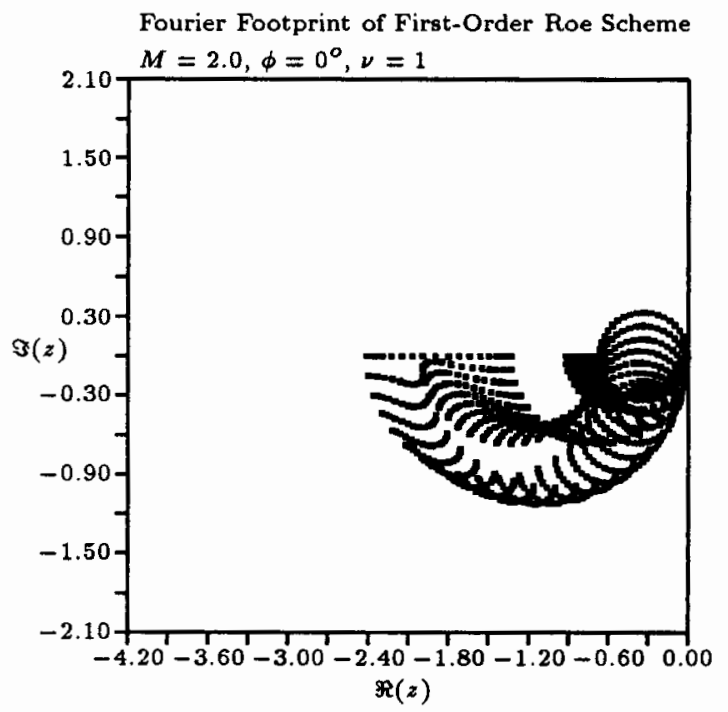

Figure 8: Fourier footprint of the first-order upwind approximation of the spatial Euler operator, for $M=2$, and flow speed aligned with the grid. 


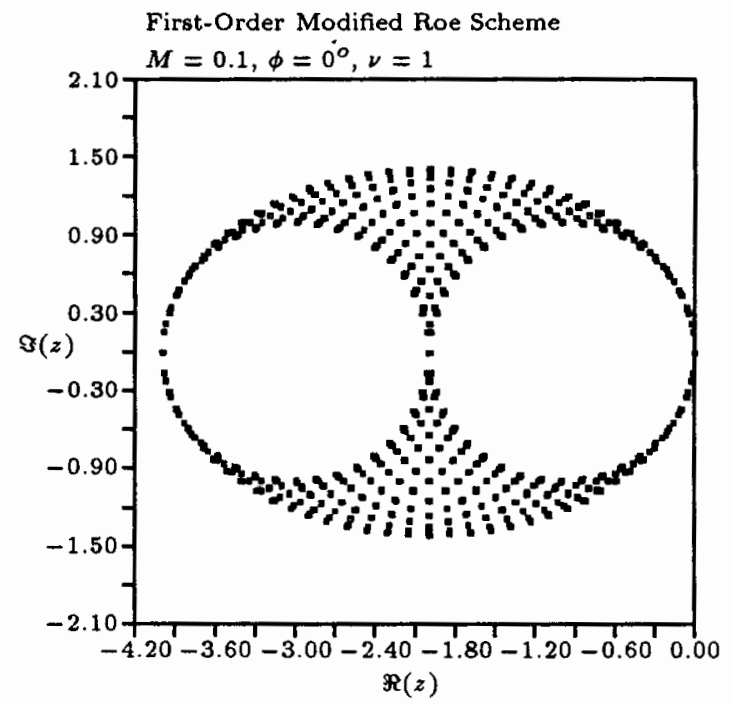

Figure 9: Fourier footprint of the preconditioned firstorder upwind approximation of the spatial Euler operator, for $M=0.1$, and flow angle $\phi=0^{\circ}$.

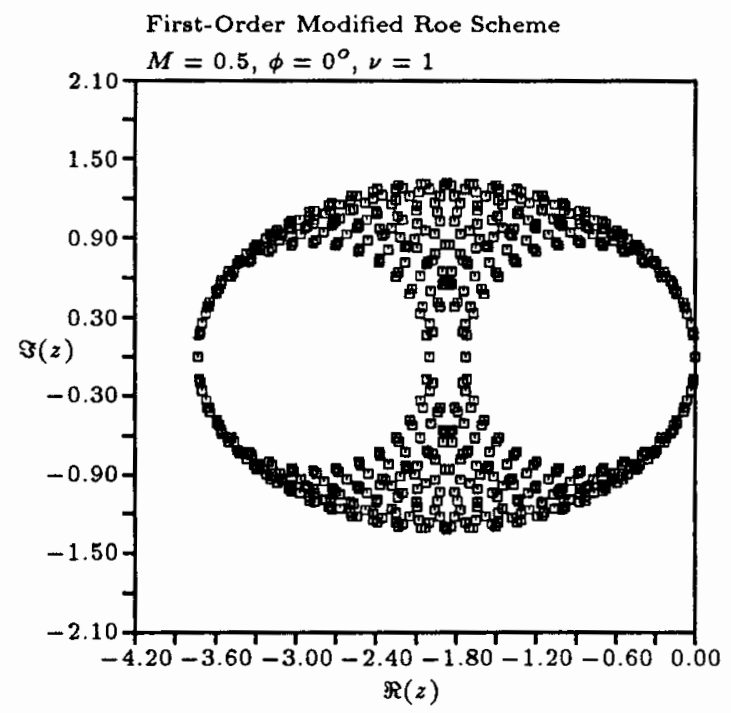

Figure 10: Fourier footprint (symbols) of the preconditioned first-order upwind approximation of the spatial Euler operator, for $M=0.5$, and flow angle $\phi=0^{\circ}$.

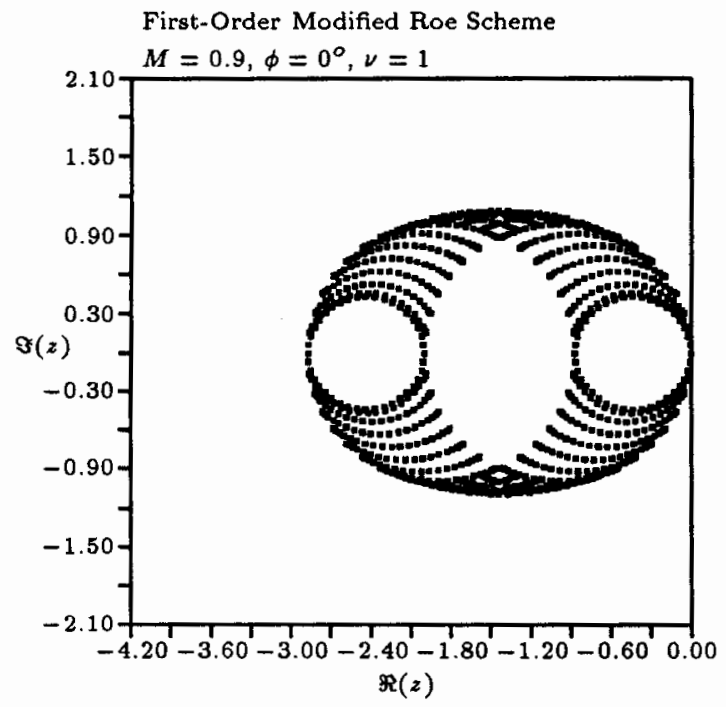

Figure 11: Fourier footprint of the preconditioned firstorder upwind approximation of the spatial Euler operator, for $M=0.9$, and flow angle $\phi=0^{\circ}$.

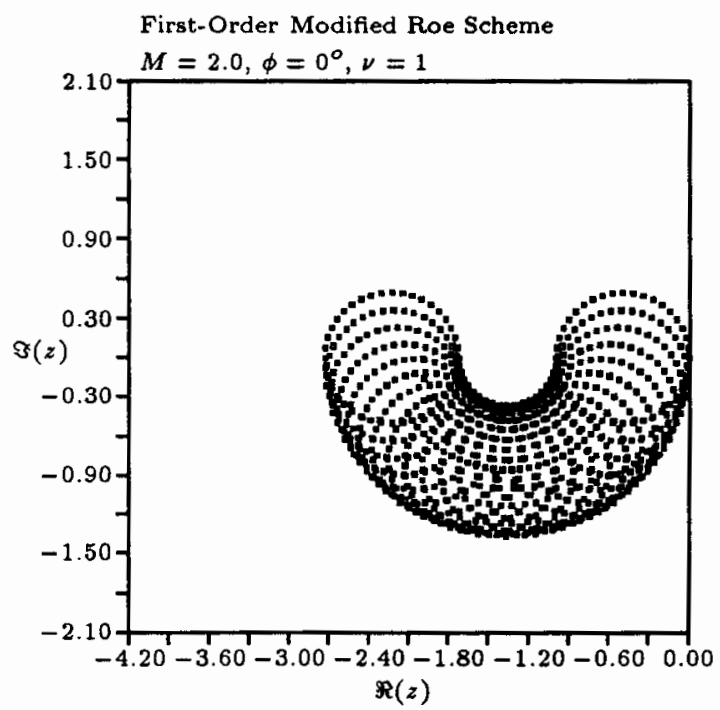

Figure 12: Fourier footprint of the preconditioned firstorder upwind approximation of the spatial Euler operator, for $M=2$, and flow angle $\phi=0^{\circ}$. 
is found. This is our functional $\sigma\left(\Delta t^{(1)}, \ldots, \Delta t^{(m)} ; M, \phi\right)$; it must be minimized by varying the $m$ parameters. (Dependence on $M$ and $\phi$ will be considered later.) The optimal (in the $L_{\infty}$ sense) $\mathrm{m}$-stage scheme may hence be obtained as the solution to the following minmax problem:

$$
\sigma_{o p t}=\min _{(\vec{\alpha}, \nu)}\left(\max _{\left|\beta_{x}\right| \text { or }\left|\beta_{y}\right| \in\left[\frac{\pi}{2}, \pi\right]}\left\|P\left(z\left(\beta_{x}, \beta_{y}, \nu\right), \vec{\alpha}\right)\right\|\right) .
$$

The optimization procedure that appeared to be most robust is Powell's method (see, e.g., [9]).

The optimization procedure is not without its problems. Specifically, there are two points of concern:

1. The alignment problem makes the optimization meaningless for flow angles near 0 (or $\pi / 2$ ), since the amplification factor for low-high (or high-low) frequency combinations tends to 1 . Our solution is to filter out these frequency combinations, e.g. for $\phi=0$ we optimize only over the eigenvalues with $\left|\beta_{y}\right| \leq\left|\beta_{x}\right|$, a wedge-like region in the frequency plane. When $\phi$ increases the wedge rotates with the flow angle and opens up, until for $\phi=\pi / 4$ the entire domain (Eqn. 7) is used. When $\phi$ increases further, the optimization domain shrinks again, until for $\phi=\pi / 2$ only those frequency pairs are included satisfying $\left|\beta_{x}\right| \leq\left|\beta_{y}\right|$. As said before, the alignment problem has to be dealt with separately, for instance by semi-coarsening $[10]$.

2. The functional has many local minima, so the optimization procedure frequently comes up with a suboptimal solution. It is important to inspect the solutions and see if they make sense; if not, the optimization algorithm must be provided with a fresh set of initial values.

To show what this technique can accomplish we present the sequence of Figures $13-16$. These include the Fourier footprint of the spatial operator, plotted on top of the level lines of the amplification factor of the multi-stage scheme that has been optimized for use with this operator. The size of the footprint scales with $\Delta t$; optimal high-frequency damping is achieved only for one particular value of $\Delta t$. Figure 13 shows, for $M=1$, $\phi=45^{\circ}$, how well the level lines can follow the outline of the footprint of the first-order upwind Euler operator when 3 stages are used. The kidney-shaped outline is due to the high-frequency entropy/shear eigenvalues; the clawshaped feature is the locus of the high-frequency acoustic eigenvalues. The value of the functional is 0.3523 , i.e., all high-frequency combinations are reduced at least by this factor. When adding one more stage (Figure 14), the attenuation is even better, namely, a factor 0.2362 ; the five-stage scheme (Figure 15) yields $\sigma=0.1652$.

Figure 16 shows how the footprint changes when the flow angle is reduced to zero: it becomes more transparent, but the outline stays the same. The level lines are those of the 4-stage scheme of Figure 14; the only change in the scheme is the adjustment of the time step (see Section 5). It is seen that the functional hardly changes $(\sigma=0.2386)$; there is no need for further optimization.

Level lines of the amplification factor in the $\left(\beta_{x}, \beta_{y}\right)$ plane are shown in Figures $17-20$ all for $M=0.1$. Data in the central square are suppressed: this is the low-frequency region. It must be understood that each frequency combination creates four eigenvalues: two are acoustic in nature, the other two correspond to entropy and shear. Each of these generates its own amplification factor. In Figure 17 , with $\phi=45^{\circ}$, for each combination of frequencies the maximum of the four amplification factors is plotted. In Figure 18 only the acoustic eigenvalues are included, while in Figure 19 only the entropy and shear-related eigenvalues are included. Finally, Figure 20 is for $\phi=0^{\circ}$, illustrating the alignment problem. Only the entropy/shear-related amplification factor is plotted; the level lines show independence of $\beta_{y}$. For $\beta_{x}=0$ the amplification factor equals 1 regardless of the $\beta_{y}$-frequency. In the optimization procedure these frequency combinations are excluded, as explained earlier. The wedge-shaped optimization region is indicated by the dashed lines.

Finally, Figures 22 - 24 show results of optimizations of schemes based on higher order upwind discretizations. Figure 22 shows the result of an optimization of a 4-stage scheme for the third-order upwind-biased Euler discretization (a $\kappa$-scheme with $\kappa=\frac{1}{3}$ [11]). The functional value is 0.6641 , which may seem rather high. However, it must be pointed out that similar functional values are obtained with the footprint of the equivalent discrete operator for the two-dimensional convection equation. Most analysis done so far was based on one-dimensional convection, which yields lower functional values for these minmax optimal solutions.

\section{Dependence on the flow angle}

As explained above, the optimization procedure generates a time-step value for which the optimal highfrequency damping is realized. For a given spatial operator and number of stages, this $\Delta t_{\text {opt }}$ depends on the Mach number and the flow angle. The variation with the flow angle is similar to that of the maximum permitted time step $\Delta t_{\max }$ for explicit convection schemes: on a square grid the stability limit drops a factor $\sqrt{2}$ when the flow angle varies from $0^{\circ}$ to $45^{\circ}$. This variation can be removed by redefining the Courant number.

For the preconditioned Euler equations, with the characteristic speeds equal to or close to $q$, we define the Courant number as

$$
\nu=q \frac{\Delta t}{l(\Delta x, \Delta y, \phi)},
$$

where $l$ is a typical cell-width that may depend on the flow direction. Figure 25 shows the typical variation of 

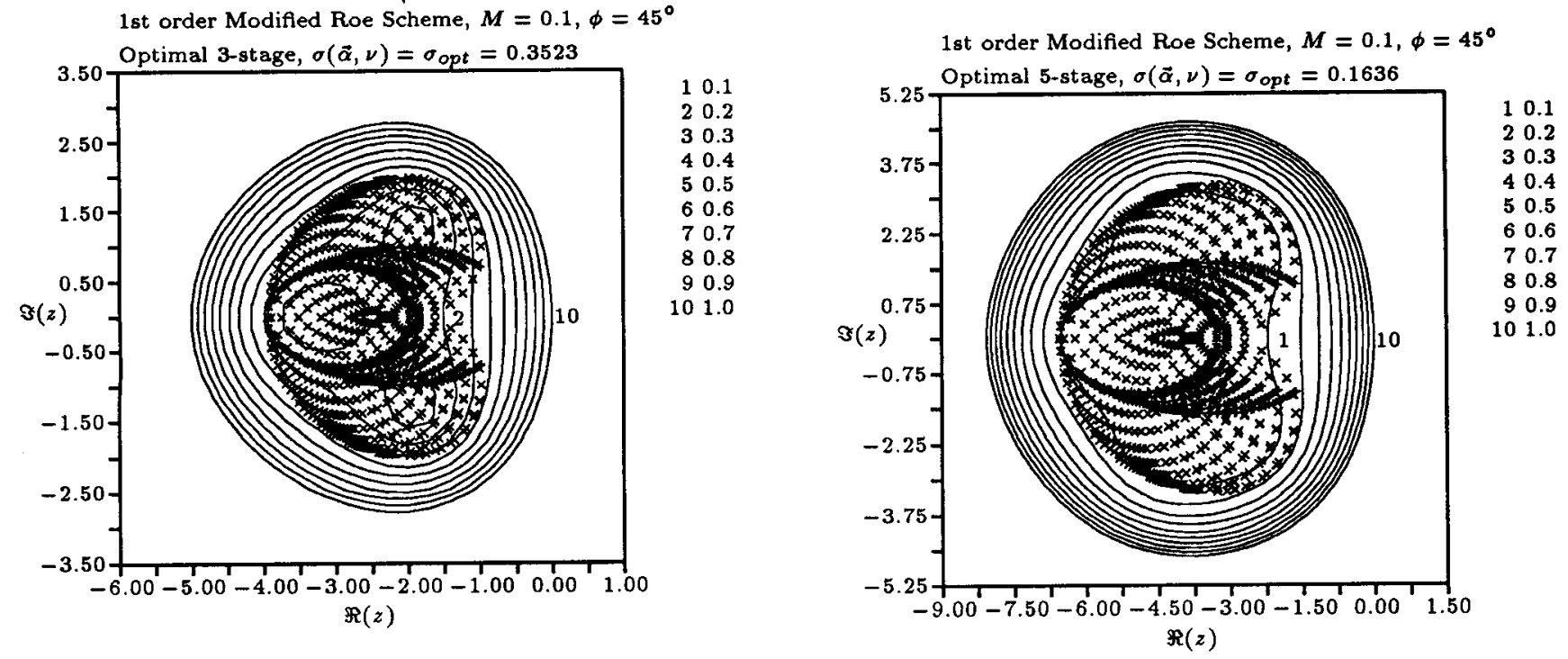

Figure 13: High-frequency Fourier footprint of the preconditioned first-order upwind Euler operator plotted on top of the level lines of the amplification factor of the associated optimal 3-stage scheme. Flow angle $45^{\circ}$.
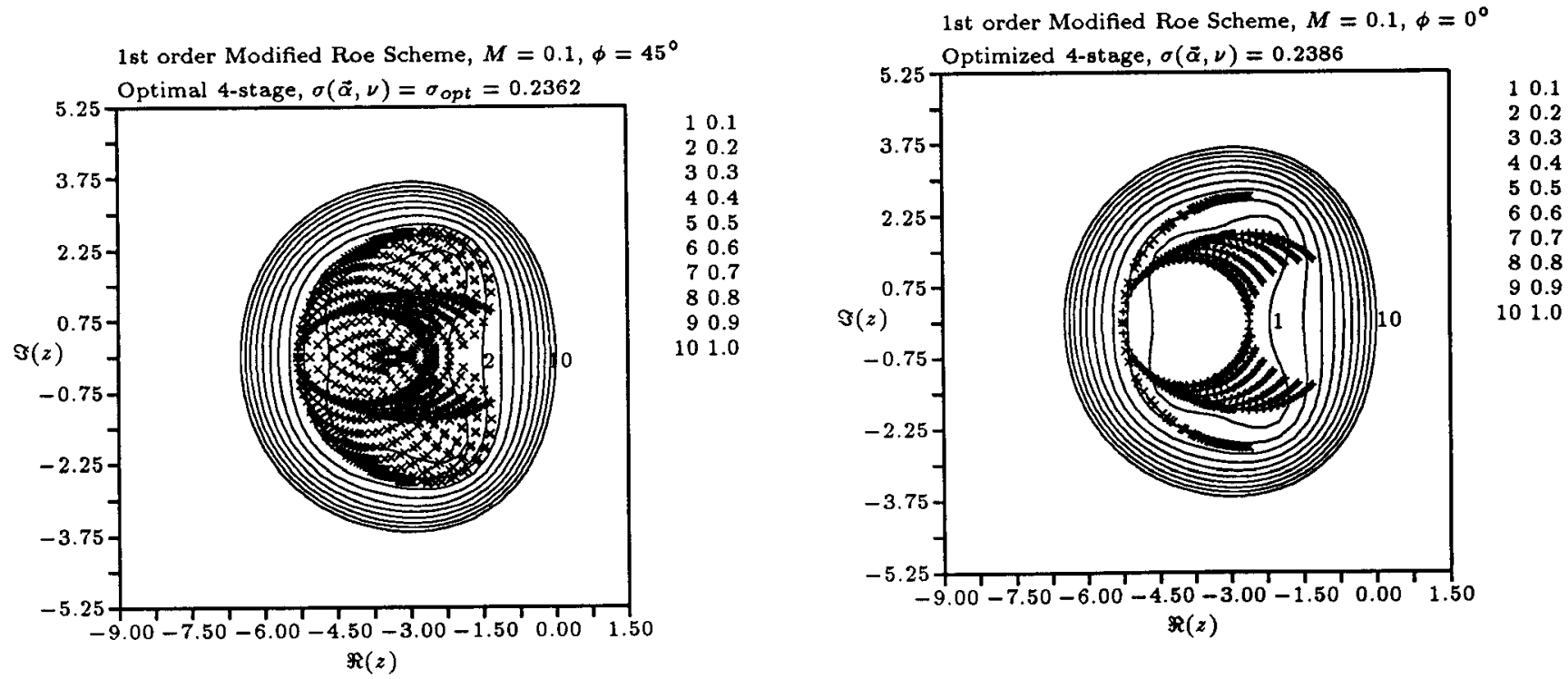

Figure 14: As Figure 13, but for optimal 4-stage scheme. 


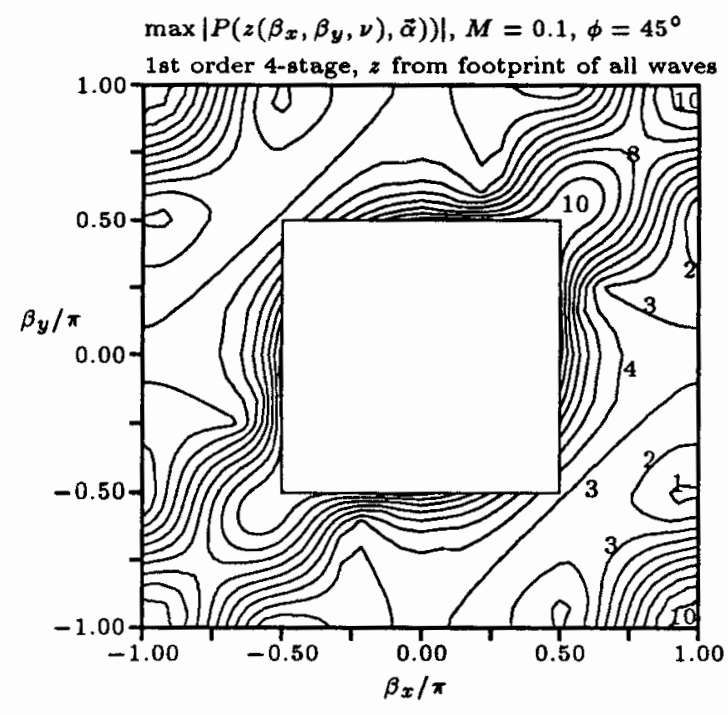

Figure 17: Level lines of the maximum amplification factor in the $\left(\beta_{x}, \beta_{y}\right)$ plane over the high-frequency domain. 1st order 4-stage scheme, $\phi=45^{\circ}$.

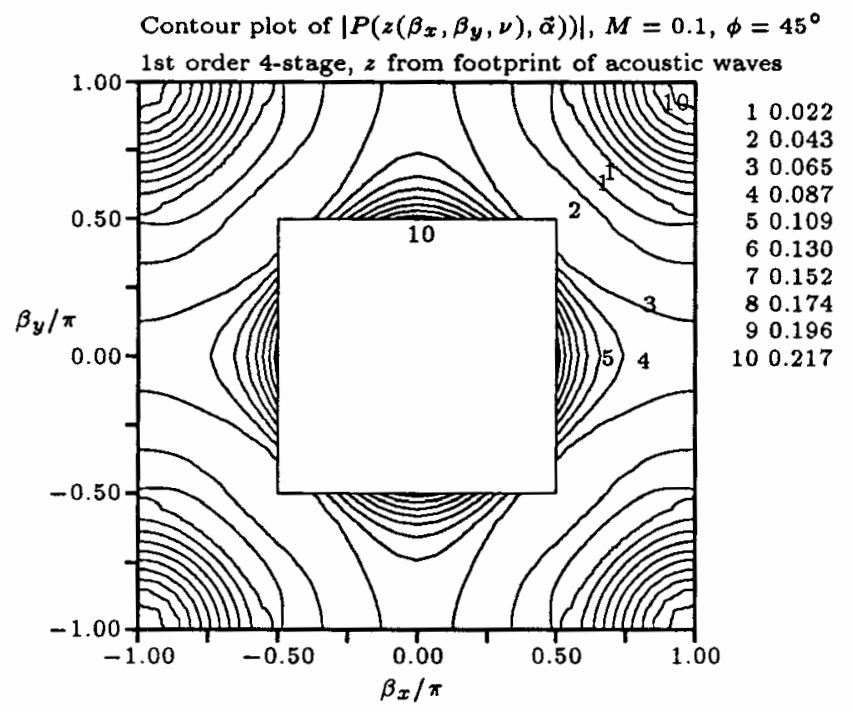

Figure 18: As Figure 17, but for acoustic waves only.

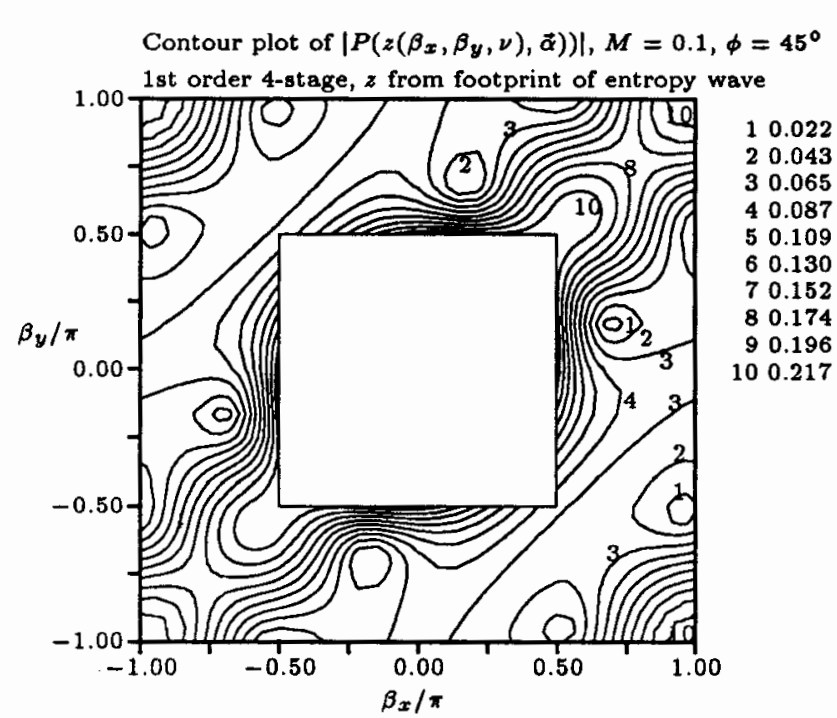

Figure 19: As Figure 17, but for shear/entropy waves only.

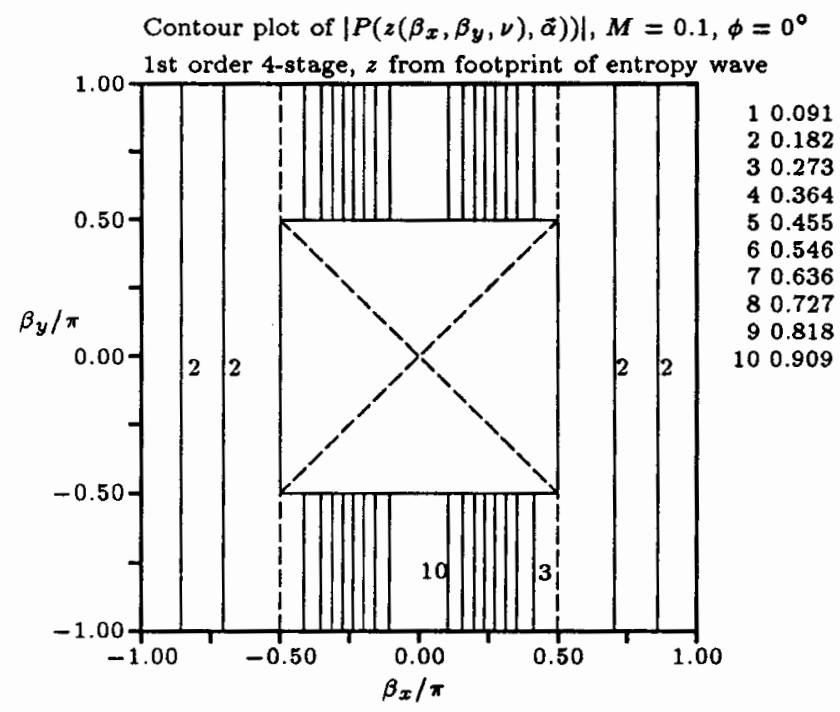

Figure 20: As Figure 19 but for flow angle $0^{\circ}$. The dashed line outlines the portion of the domain considered. 


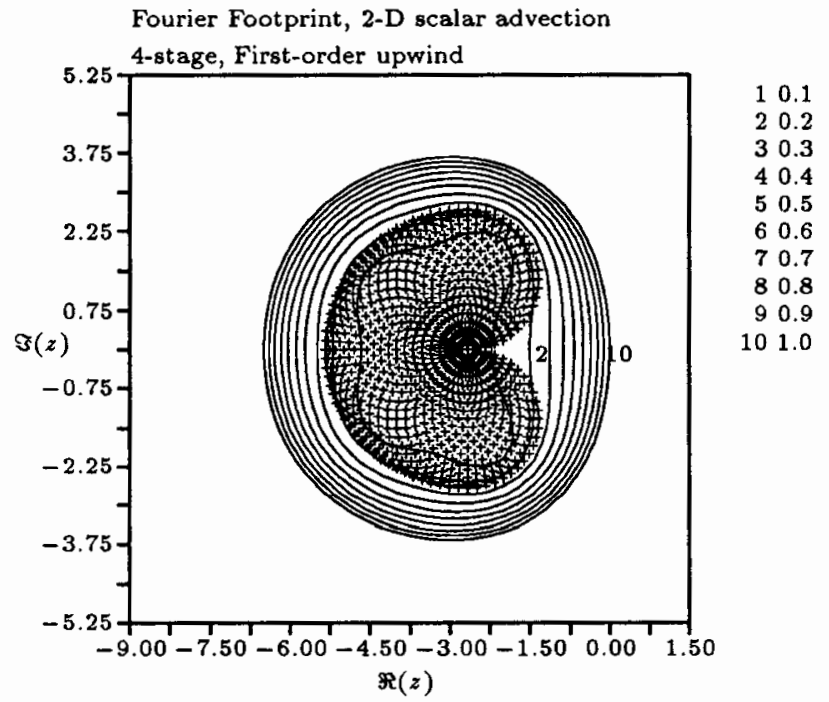

Figure 21: High-frequency Fourier footprint of the firstorder upwind 2-D scalar advection operator plotted on top of the level lines of the amplification factor of the 4-stage scheme optimized using the Modified Roe operator for the Euler equations. Convection angle $45^{\circ}$.

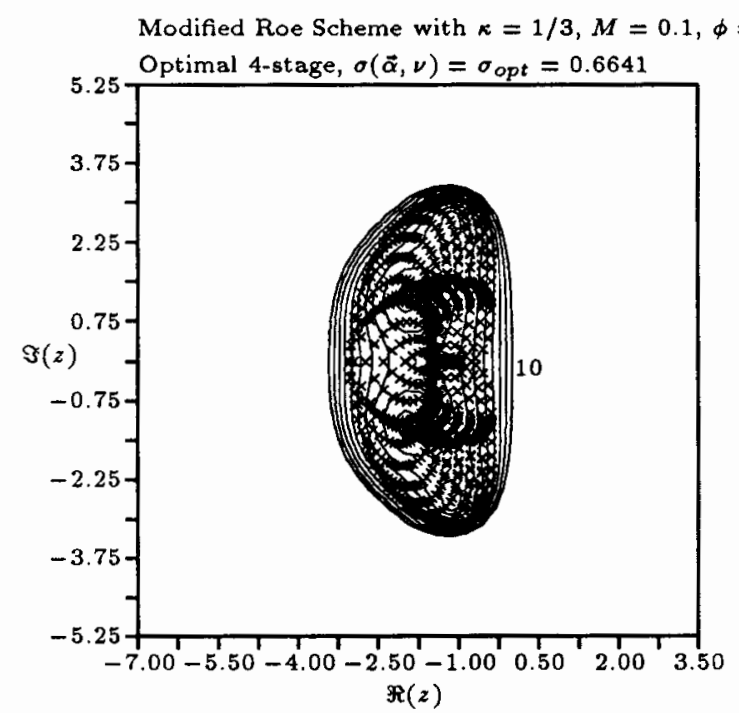

Figure 22: High-frequency Fourier footprint of the preconditioned third-order upwind Euler operator plotted on top of the level lines of the amplication factor of the associated optimal 4-stage scheme.

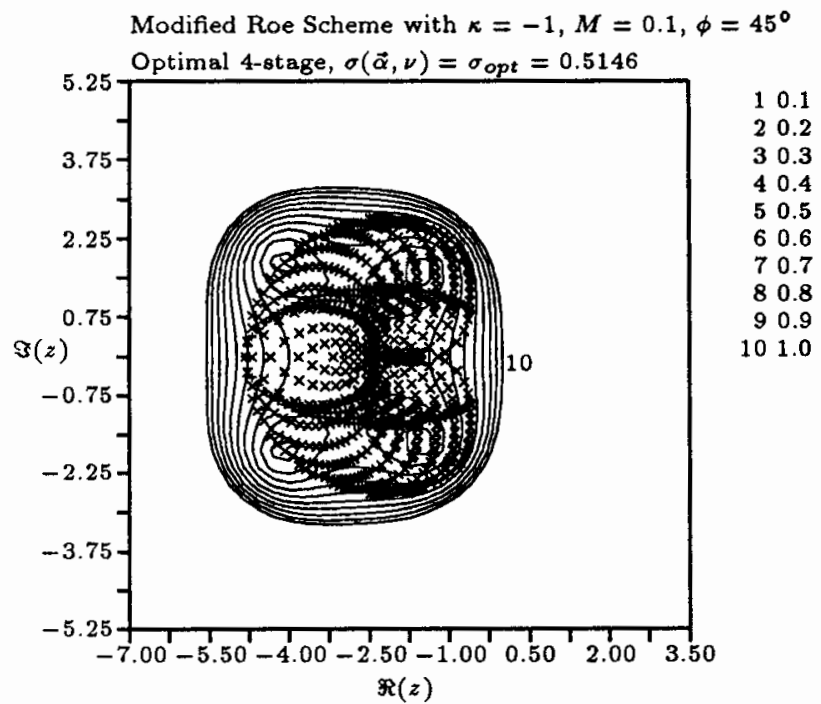

Figure 23: High-frequency Fourier footprint of the preconditioned second-order $(\kappa=-1)$ upwind Euler operator plotted on top of the level lines of the amplication factor of the associated optimal 4-stage scheme.

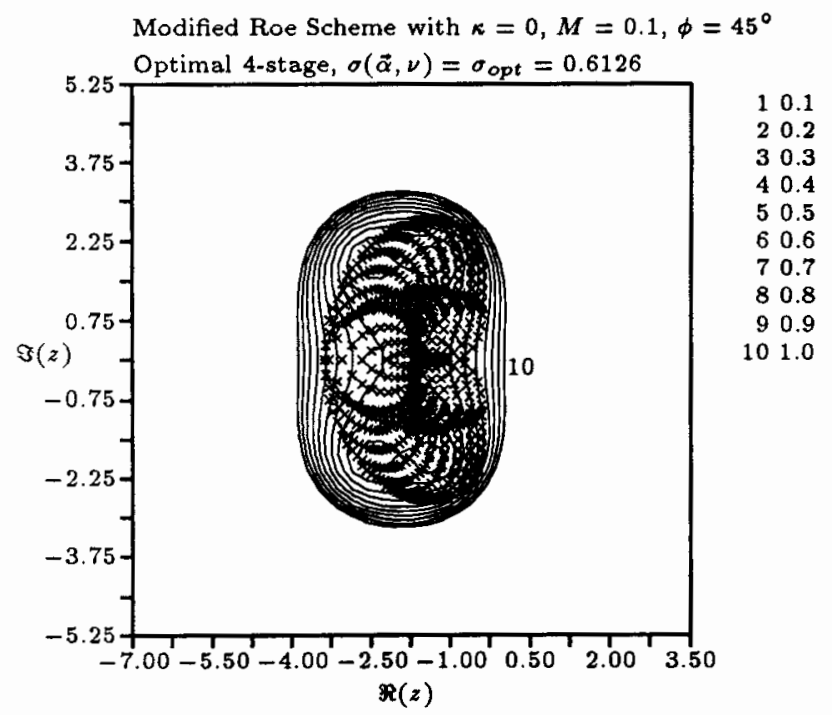

Figure 24: High-frequency Fourier footprint of the preconditioned second-order $(\kappa=0)$ upwind Euler operator plotted on top of the level lines of the amplication factor of the associated optimal 4-stage scheme. 
$\nu_{\text {opt }}$ with the flow angle for a square grid, using a fixed $l=\Delta x=\Delta y$. For small $\phi$ the optimal value of the Courant number is dictated by the size of the acoustic footprint; between $10^{\circ}$ and $15^{\circ}$ the entropy/shear footprint takes over. (This feature is a function of the wedge filter in the frequency domain, described in the previous section.) The curves for different Mach numbers are very close, except when $M$ approaches 1 ; for comparison we have also plotted the function $\cos \phi+\sin \phi$, which represents the length of the projection of the cell diagonal on the streamline. For general rectangular cells we find that defining

$$
l=\Delta x|\cos \phi|+\Delta y|\sin \phi|
$$

takes away most of the variation of $\nu_{\text {opt }}$ with the flow angle, so that a single value can be recommended.

\section{Dependence on the Mach num- ber}

Figures 26 and 27 show the result of optimizing the 4-stage scheme for higher Mach numbers than before, viz. $M=0.5$ and $M=0.9$; the flow angle is $0^{\circ}$. The acoustic footprint bears evidence of a growing disparity among the characteristic speeds: acoustic waves traveling in the flow direction only move at a speed $q \sqrt{1-M^{2}}$, while normal to the flow direction the propagation speed still equals $q$. The smaller speed moves a group of high-frequency eigenvalues toward the origin, causing higher functional values for a given number of stages. In comparison to Figure 27 it is seen that $\sigma$ is increased only slightly (to 0.2604 ) for $M=0.5$, but significally (to 0.4100 ) for $M=0.9$.

It was our goal, given the spatial differencing operator and the number of stages, to produce a single set of multi-stage parameters that yield effective high-frequency damping for any flow angle or Mach number. As explained in the previous section, the influence of the flow angle is minor, once the alignment problem has been removed. By the same token, the influence of the Mach number is minor once we recognize there is no remedy for the deterioration of high-frequency damping as $M$ approaches 1 , other than using more stages. If we give the multi-stage parameters a weight $\sqrt{1-\min \left(M^{2}, M^{-2}\right)}$, the sonic problem is removed, and a useful set of parameters can be chosen.

\section{Optimal multi-stage schemes}

We have computed optimal multi-stage schemes (Tables 1 - 4) based on the Modified Roe discrete operator for the preconditioned Euler equations. Though these coeffcients have been computed based on this specific operator, it is expected that they will be useful with other discrete spatial operators for the preconditioned Euler and NavierStokes equations as well.

These schemes are not only preferable as solvers in a multi-grid strategy, but are also superior single-grid
Number of Stages

\begin{tabular}{|c||c|c|c|c||}
\hline & 2 & 3 & 4 & 5 \\
\hline$\alpha_{1}$ & 0.3019 & 0.1387 & 0.0786 & 0.0510 \\
\hline$\alpha_{2}$ & 1 & 0.3932 & 0.1999 & 0.1221 \\
\hline$\alpha_{3}$ & & 1 & 0.4224 & 0.2326 \\
\hline$\alpha_{4}$ & & & 1 & 0.4402 \\
\hline$\alpha_{5}$ & & & & 1 \\
\hline$\nu$ & 0.9822 & 1.3953 & 1.8881 & 2.3102 \\
\hline$\sigma_{\text {opt }}$ & 0.5521 & 0.3523 & 0.2362 & 0.1652 \\
\hline
\end{tabular}

Table 1: Multi-stage Coefficients for Optimal First-Order Scheme.

Number of Stages

\begin{tabular}{|c||c|c|c|c||}
\hline & 2 & 3 & 4 & 5 \\
\hline$\alpha_{1}$ & 0.5379 & 0.2178 & 0.1288 & 0.0807 \\
\hline$\alpha_{2}$ & 1 & 0.5360 & 0.2677 & 0.1801 \\
\hline$\alpha_{3}$ & & 1 & 0.5347 & 0.3019 \\
\hline$\alpha_{4}$ & & & 1 & 0.5391 \\
\hline$\alpha_{5}$ & & & & 1 \\
\hline$\nu$ & 0.5744 & 0.9383 & 1.1991 & 1.5192 \\
\hline$\sigma_{\text {opt }}$ & 0.7951 & 0.6842 & 0.6126 & 0.5316 \\
\hline
\end{tabular}

Table 2: Multi-stage Coefficients for Optimal $\kappa=0$ Scheme.

\begin{tabular}{|c||c|c|c|c||}
\hline \multicolumn{7}{|c||}{ Number of Stages } \\
\hline$\alpha_{1}$ & 0.4222 & 0.1597 & 0.0884 & 0.0567 \\
\hline$\alpha_{2}$ & 1 & 0.4304 & 0.2227 & 0.1398 \\
\hline$\alpha_{3}$ & & 1 & 0.4864 & 0.2639 \\
\hline$\alpha_{4}$ & & & 1 & 0.5017 \\
\hline$\alpha_{5}$ & & & & 1 \\
\hline$\nu$ & 0.3736 & 0.7018 & 0.8503 & 1.0352 \\
\hline$\sigma_{\text {opt }}$ & 0.7723 & 0.6614 & 0.5146 & 0.4357 \\
\hline
\end{tabular}

Table 3: Multi-stage Coefficients for Optimal $\kappa=-1$ Scheme. 
Number of Stages

\begin{tabular}{|c||c|c|c|c||}
\hline & 2 & 3 & 4 & 5 \\
\hline$\alpha_{1}$ & 0.6807 & 0.2781 & 0.1624 & 0.1154 \\
\hline$\alpha_{2}$ & 1 & 0.5212 & 0.2755 & 0.2055 \\
\hline$\alpha_{3}$ & & 1 & 0.5025 & 0.3330 \\
\hline$\alpha_{4}$ & & & 1 & 0.5566 \\
\hline$\alpha_{5}$ & & & & 1 \\
\hline$\nu$ & 0.6723 & 1.1263 & 1.6073 & 1.8232 \\
\hline$\sigma_{\text {opt }}$ & 0.8262 & 0.7463 & 0.6641 & 0.5994 \\
\hline
\end{tabular}

Table 4: Multi-stage Coefficients for Optimal $\kappa=1 / 3$ Scheme.

schemes, as the preconditioning itself already accelerates the convergence to a steady solution, and the highfrequency damping provides robustness.

\section{Multi-grid convergence studies}

With reference to the convergence plots (Figures 28 - 35), the key prec refers to the use of the Modified Roe discretization along with coefficients derived in this paper. no prec refers to Roe's scheme along with Tai's coefficients, $\mathbf{m g}$ indicates that the line is the result of using 4 grid levels. (A Sawtooth-FAS cycle was used in these multi-grid calculations.) A work unit is defined as the work required for a single stage relaxation sweep (update) on the fine grid. Since the relaxation schemes vary, this would seem inconsistent. However, if coded properly, the preconditioned scheme is comparable in cost to the regular scheme.

A first test case was the computation of steady flow over a NACA 0012 airfoil at Mach number 0.63 and angle of attack $2^{\circ}$ on a $32 \times 16$ O-grid. Residual convergence histories, with and without preconditioning on single- and multi-grids, are presented in Figure 35. It is seen that preconditioning and multi-grid marching accelerate the convergence independently. The multi-grid technique is more effective for the preconditioned scheme than for the standard scheme because of the superior damping properties of the preconditioned scheme, i.e. the speed-up should be greater for the preconditioned scheme. This has been demonstrated by Tai [6] for one-dimensional Euler computations (see Table 5). Evidence of this speed-up in the two-dimensional case is demonstrated in Figure 35. A speed-up factor of 2.73 over the standard scheme is obtained by using multi-grid with the standard scheme and the corresponding speed-up factor with multi-grid over the preconditioned scheme is 3.21 . The corresponding gain by using multi-grid with preconditioning over traditional multi-grid is a speed-up factor of 1.6 for this case. The
Variation of Courant number with flow angle. 1st order Modified Roe spatial operator

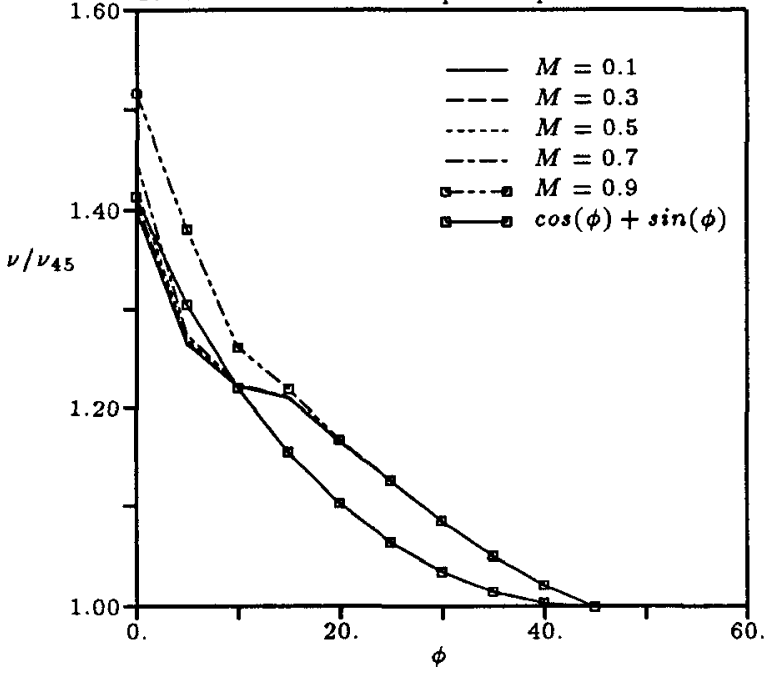

Figure 25: Variation of the optimal Courant number with the flow angle, for the first-order upwind 4-stage scheme.

two-dimensional case is qualitatively different because of the appearance of stagnation points, which cause trouble in the preconditioning step. In order to reap the full multi-grid benefits it is necessary to make the preconditioning scheme more robust near flow singularities; this is the focus of a parallel research effort.

Another test case considered was the propagation of a small pressure disturbance (Euler) in a square domain. Figures 28 to 34 give the convergence histories for the calculation of the propogation of a $2.0 \%$ pressure disturbance on a square $32 \times 32$ mesh domain. The multi-grid computations made use of 4 levels. In these cases, the damping capabilities of the multi-stage schemes can be more easily observed, since it is not necessary to employ smoothing at the problem "points" for the preconditioning step $(M \rightarrow 0$ and $M \uparrow 1$ ), something that may affect damping.

A pressure disturbance decomposes into acoustic waves and an entropy wave (a nonlinear disturbance). The condition number for local time-stepping equals $(M+$ $1) / \min (M,|M-1|)$, and has a theoretical minimum at $M=0.5$. Indeed, as Figure 29 shows, local time-stepping performs best at this Mach number and multi-grid convergence with local time-stepping is as good as with characteristic time-stepping for this case.

In general, however, multi-grid with matrix preconditioning is observed to be the most efficient of the schemes compared, both for first-order and second-order upwind discretizations. As expected, there is a Mach number dependence in the speed-ups observed. Large speed-ups with the preconditioned schemes are observed where the condition number for local time-stepping is high (Figures 28, $30,32$ and 34$)$. 


\begin{tabular}{|c|c|c|c|c|c|c|c|c|c|c|c|}
\hline \multicolumn{12}{|c|}{ Number of stages } \\
\hline & & \multicolumn{5}{|c|}{ Local time-stepping } & \multicolumn{5}{|c|}{ Matrix preconditioning } \\
\hline \multirow[b]{2}{*}{ Number } & & 2 & 3 & 4 & 5 & 6 & 2 & 3 & 4 & 5 & 6 \\
\hline & 1 & 6455 & 5396 & 5161 & 4952 & 4826 & 2432 & 1968 & 1881 & 1808 & 1760 \\
\hline \multirow{2}{*}{$\begin{array}{l}\text { of } \\
\text { grid }\end{array}$} & 2 & 1135 & 1277 & 1441 & 1580 & 1719 & 376 & 449 & 517 & 573 & 632 \\
\hline & 3 & 822 & 844 & 890 & 938 & 985 & 170 & 202 & 235 & 270 & 303 \\
\hline \multirow[t]{3}{*}{ levels } & 4 & 795 & 783 & 804 & 832 & 875 & 140 & 144 & 157 & 172 & 182 \\
\hline & 5 & 871 & 802 & 800 & 797 & 882 & 150 & 146 & 159 & 180 & 183 \\
\hline & 6 & 632 & 589 & 625 & 648 & 718 & 243 & 222 & 218 & 238 & 264 \\
\hline
\end{tabular}

Table 5: Work required for convergence for the calculation of shockless transonic flow in a converging-diverging channel. The basic grid has 256 cells, the spatial discretization is third-order upwind-biased. Using Tai's multi-stage parameters, the preconditioned scheme not only accelerates convergence on a single grid, but also yields a greater multi-grid benefit than the standard scheme, which merely uses a local time step. The unit used here is the number of iterations needed to reduce the residual norm from around $10^{-2}$ to around $10^{-10}$.

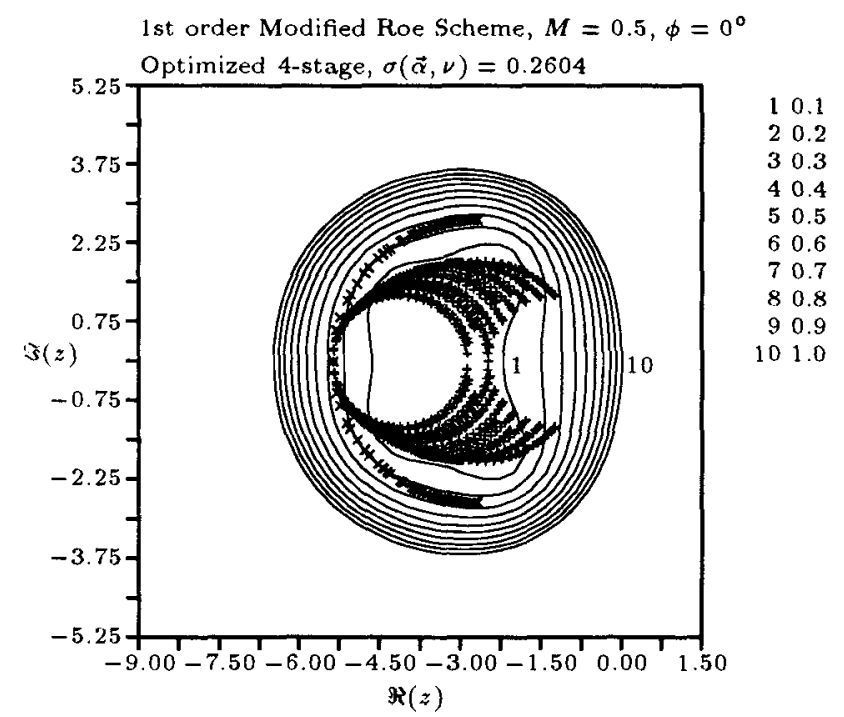

Figure 26: High-frequency Fourier footprint of the preconditioned first-order upwind Euler operator plotted on top of the level lines of the amplification factor of the associated optimal 4-stage scheme. Flow angle $0^{\circ}, M=0.5$.

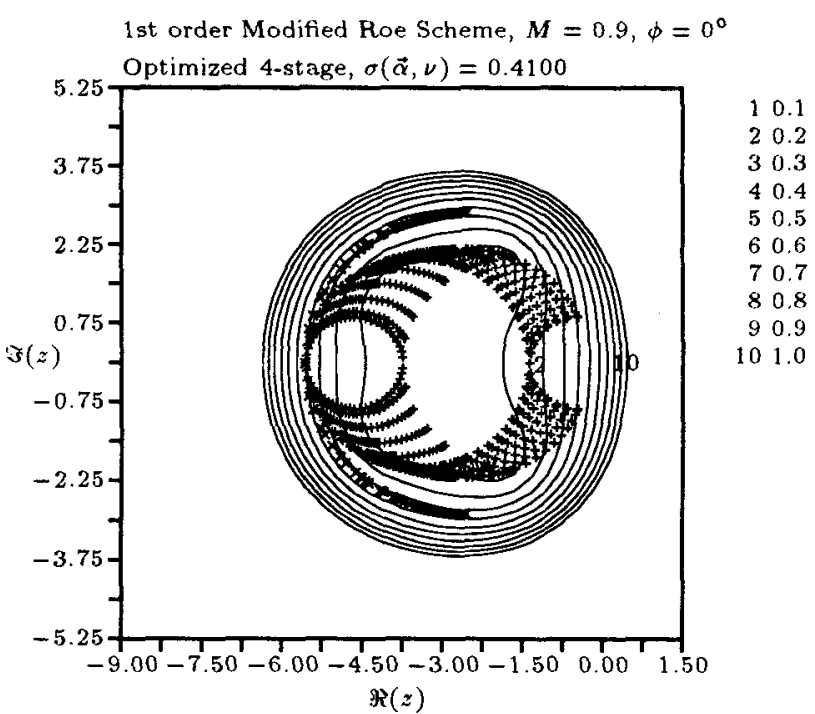

Figure 27: High-frequency Fourier footprint of the preconditioned first-order upwind Euler operator plotted on top of the level lines of the amplification factor of the associated optimal 4-stage scheme. Flow angle $0^{\circ}, M=0.9$. 


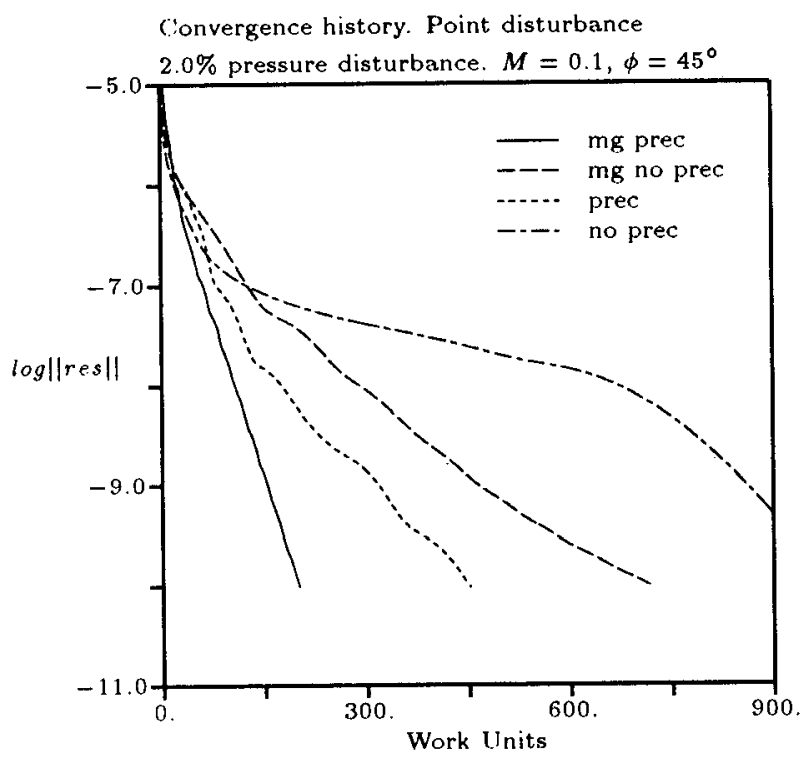

Figure 28: Convergence histories for calculations of the propagation of a pressure disturbance. The optimal firstorder upwind 3-stage scheme is used with local timestepping (Tai) or matrix preconditioning (new coefficients), in single- or 4-grid relaxation.

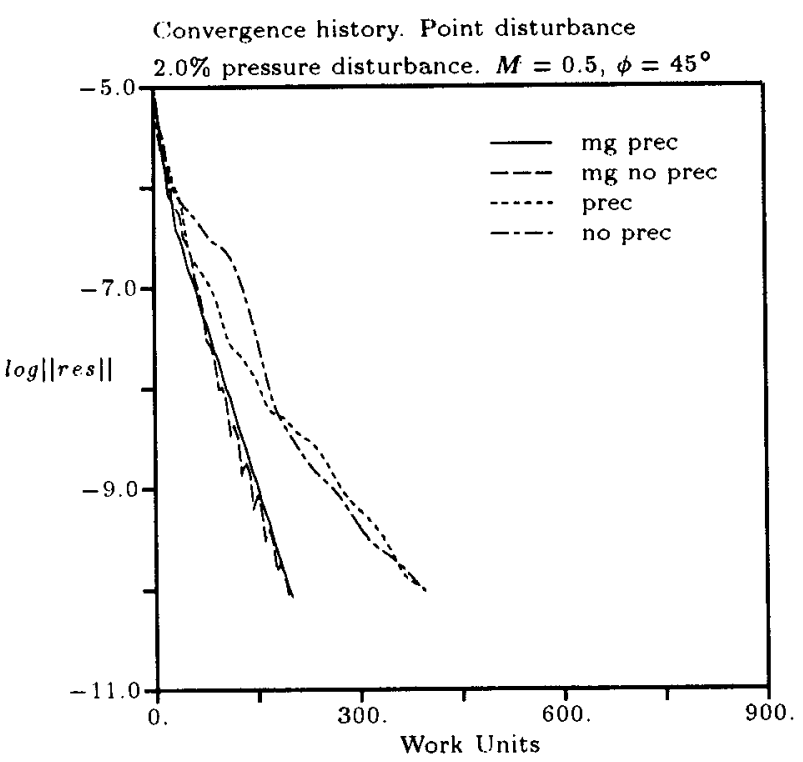

Figure 29: Convergence histories for calculations of the propagation of a pressure disturbance. As in Figure 28, but with $M=0.5$.

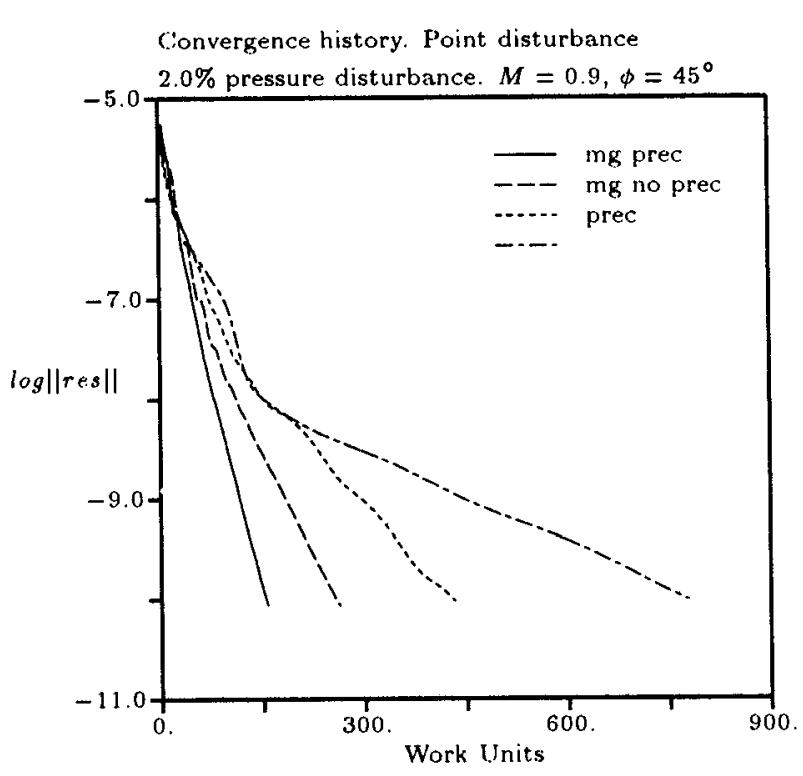

Figure 30: Convergence histories for calculations of the propagation of a pressure disturbance. As in Figure 28, but with $M=0.9$.

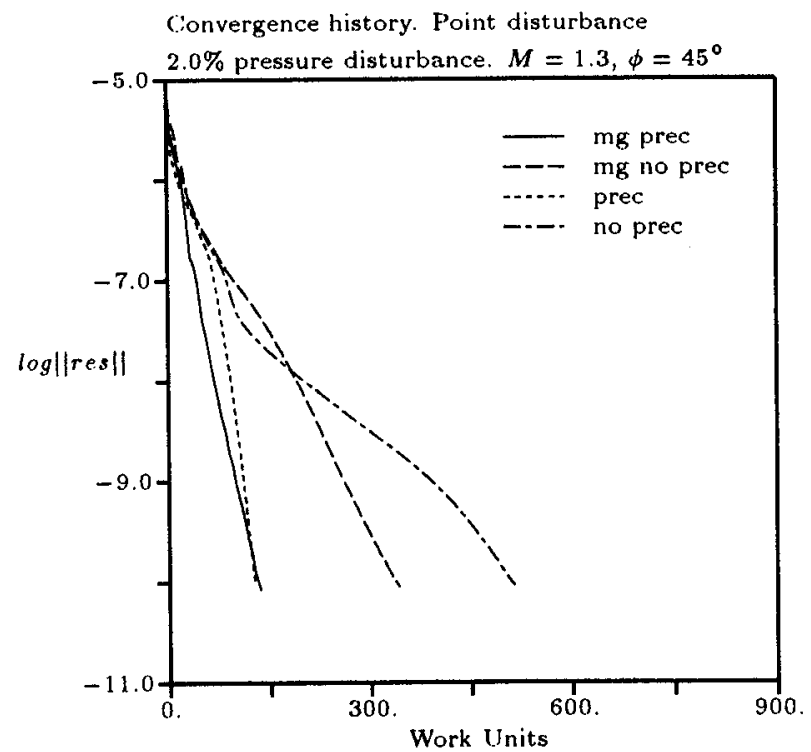

Figure 31: Convergence histories for calculations of the propagation of a pressure disturbance. As in Figure 28, but with $M=1.3$. 


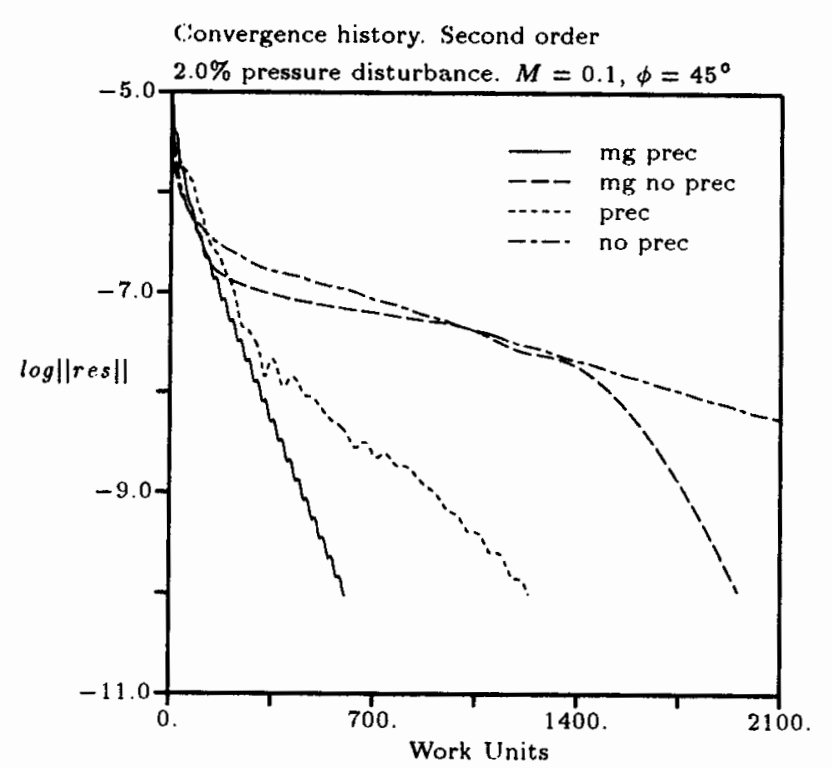

Figure 32: (onvergence histories for calculations of the propagation of a pressure disturbance. The optimal second-order upwind $(\kappa=-1) 3$-stage scheme is used with local time-stepping (Tai) or matrix preconditioning (new coefficients), in single- or 4-grid relaxation.

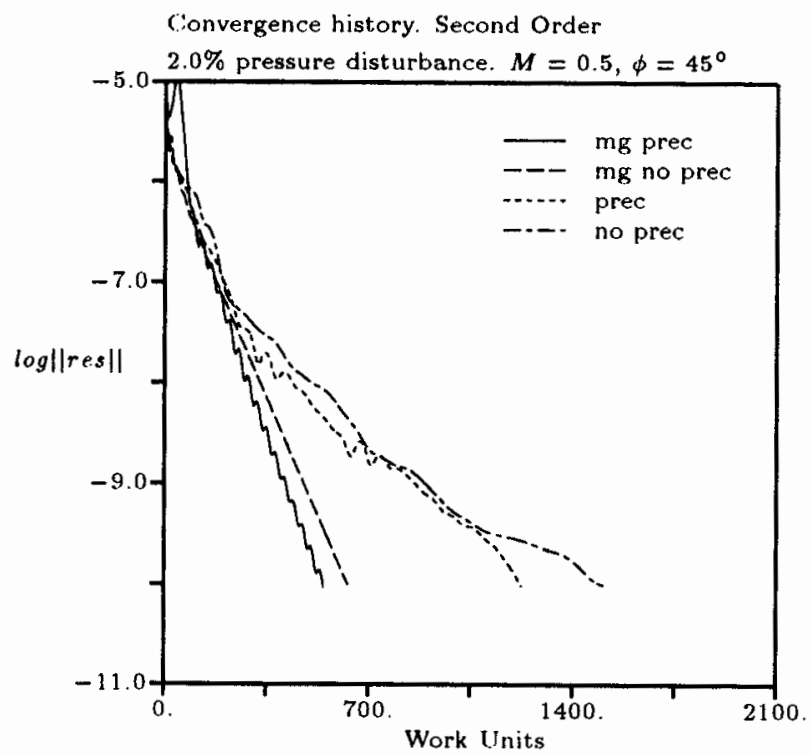

Figure 33: Convergence histories for calculations of the propagation of a pressure disturbance. As in Figure 32, but with $M=0.5$.

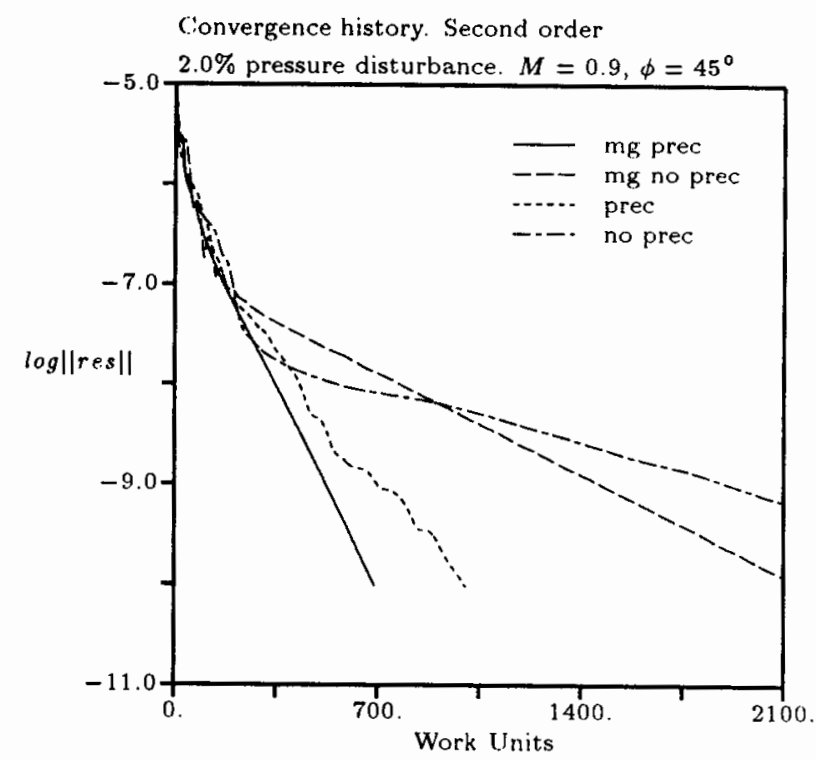

Figure 34: Convergence histories for calculations of the propagation of a pressure disturbance. As in Figure 32, but with $M=0.9$.

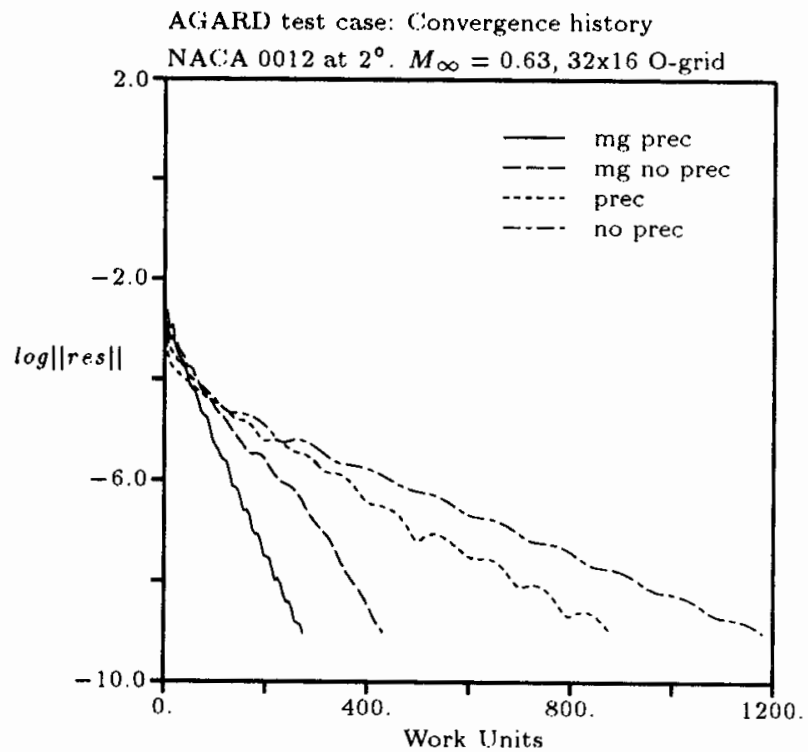

Figure 35: Convergence histories for calculations of the flow over a NAC;A 0012 airfoil, with $\alpha=0^{\circ}, M=0.63$, on a $32 \times 16 \mathrm{O}$-grid. The optirnal first-order upwind 3-stage scheme is used with local time-stepping (Tai) or matrix preconditioning (new coefficients), in single- or 4-grid relaxation. 


\section{Optimization of Navier-Stokes schemes}

If the proper preconditioning is used (see the companion paper [7]), Navier-Stokes discretizations do not offer any extra difficulty - in theory. With decreasing cell Reynold-number the footprints get more and more concentrated near the real axis, where damping rates are excellent anyway; the natural viscosity causes high frequency eigenvalues to move away from the origin along the negative real axis, which is helpful. The influence of the cell aspect-ratio is removed by appropriate scaling of elements in the preconditioning matrix.

Thus, it appears that for a given spatial NavierStokes discretization and a given number of stages, a set of multi-stage parameters can be produced that provide superior high-frequency damping for all flow angles, Mach numbers, cell Reynolds numbers and cell aspect-ratios.

\section{Concluding Remarks}

In this paper, we have describe an optimization technique that can be used to obtain multi-stage schemes that optimally damp high-frequency waves admitted by a particular preconditioned Euler or Navier-Stokes discrete operator. We have also tabulated a set of these coefficients based on a first-order upwind discretization and some higher order $(\kappa=0,-1,1 / 3)$ upwind discretizations of the Euler equations. Though these coefficients are based on a particular discrete operator (Modified Roe), it is expected that they will be useful with other discrete operators for the preconditioned Euler and Navier-Stokes as well, for use in both a multi-grid and single-grid context.

Results have been presented for some test cases. These results indicate that multi-grid with matrix preconditioning is more efficient than multi-grid with local time-stepping, in general, and that multi-grid and matrix preconditioning accelerate the convergence to a steady solution individually.

Further work is required to make the airfoil code (matrix preconditioning with Modified Roe scheme) more robust. We are also beginning multi-grid studies of NavierStokes cases.

\section{Acknowledgements}

This work was funded by the Boeing (Commercial Airplane Company, monitored by Dr. Laurence Wigton.

\section{References}

[1] B. van Leer, W. T. Lee, and P. L. Roe, "Characteristic time-stepping or local preconditioning of the Euler equations," in AIAA 10th Computational Fluid Dynamics Conference, 1991.
[2] A. Jameson, "Numerical solution of the Euler equations for compressible inviscid fluids," in Numerical Methods for the Euler Equations of Fluid Dynamics (F. Angrand, A. Dervieux, J. A. Désidéri, and R. ('lowinski, eds.), SIAM, 1985.

[3] B. van Leer, C. H. Tai, and K. (X. Powell, "Design of optimally-smoothing multi-stage schemes for the Euler equations," in AIAA 9th Computational Fluid Dynamics Conference, 1989.

[4] L. A. Catalano and H. Deconinck, "Two-dimensional optimization of smoothing properties of multi-stage schemes applied to hyperbolic equations," in Proceedings of the Third European Conference on Multigrid Methods, 1990.

[5] B. van Leer, W. T. Lee, P. L. Roe, K. (A. Powell, and C. H. Tai, "Design of optimally-smoothing schemes for the Euler equations," Journal of Applied and $\mathrm{Nu}$ merical Mathematics, 1991.

[6] (G.-H. Tai, Acceleration Techniques for Explicit Euler Codes. PhD thesis, University of Michigan, 1990.

[7] D. Lee and B. van Leer, "Progress in local preconditioning of the Euler and Navier-Stokes equations," in AIAA 11th Computational Fluid Dynamics Conference, 1993.

[8] P. L. Roe, "Characteristic-based schemes for the Euler equations," Annual Review of Fluid Mechanics, vol. 18, pp. 337-365, 1986.

[9] R. P. Brent, Algorithms for Minimization without Derivatives. Prentice-Hall, Englewood Cliffs, N.J., 1973.

[10] W. A. Mulder, "A new multigrid approach to convection problems," CAM Report 88-04, UCLA Computational and Applied Mathematics, 1988.

[11] W. K. Anderson, J. L. Thomas, and B. van Leer, "A comparison of finite volume flux vector splittings for the Euler equations," AIAA Journal, vol. 24, 1985. 